\title{
Quenching the Chiral Magnetic Effect via the Gravitational Anomaly and Holography
}

\author{
Karl Landsteiner, ${ }^{*}$ Esperanza Lopez, ${ }^{\dagger}$ and Guillermo Milans del Bosch ${ }^{\ddagger}$ \\ Instituto de Física Teórica UAM/CSIC, C/ Nicolás Cabrera 13-15, Cantoblanco, 28049 Madrid, Spain
}

(Received 29 September 2017; revised manuscript received 16 January 2018; published 13 February 2018)

\begin{abstract}
In the presence of a gravitational contribution to the chiral anomaly, the chiral magnetic effect induces an energy current proportional to the square of the temperature in equilibrium. In holography the thermal state corresponds to a black hole. We numerically study holographic quenches in which a planar shell of scalar matter falls into a black hole and raises its temperature. During the process the momentum density (energy current) is conserved. The energy current has two components, a nondissipative one induced by the anomaly and a dissipative flow component. The dissipative component can be measured via the drag it asserts on an additional auxiliary color charge. Our results indicate strong suppression very far from equilibrium.
\end{abstract}

DOI: 10.1103/PhysRevLett.120.071602

Anomaly induced transport phenomena have been in the focus of much research in recent years [1,2]. The prime example is the so called chiral magnetic effect (CME) [3]. The CME has a component due to the gravitational contribution to the chiral anomaly. Chiral fermions in the background of gauge and gravitational fields have the anomaly

$$
\partial_{\mu} J^{\mu}=\varepsilon^{\mu \nu \rho \sigma}\left(\alpha F_{\mu \nu} F_{\rho \sigma}+\lambda R_{b \mu \nu}^{a} R_{a \rho \sigma}^{b}\right) .
$$

In the presence of a magnetic field $\vec{B}$ and at temperature $T$ chiral fermions build up an energy current $[4,5]$

$$
\vec{J}_{\varepsilon}=32 \pi^{2} T^{2} \lambda \vec{B}
$$

In holography the gravitational anomaly is implemented via a mixed Chern-Simons term. Space-time is curved in the additional holographic direction and this is what generates Eq. (2) from the mixed Chern-Simons term [6]. Perturbative nonrenormalization has been shown in Refs. [7,8]. The relation of Eq. (2) with the gravitational contribution to the chiral anomaly has also been derived in a model independent manner combining hydrodynamic and geometric arguments in Refs. [9,10]. An additional constraint on effective actions consistent with Eq. (2) stems from considerations based on global gravitational anomalies $[11,12]$. Transport signatures induced by Eq. (2) have recently been reported in the Weyl semimetal $\mathrm{NbP}$ [13].

So far anomaly induced transport has mostly been studied in a near equilibrium setup in which local versions of

Published by the American Physical Society under the terms of the Creative Commons Attribution 4.0 International license. Further distribution of this work must maintain attribution to the author(s) and the published article's title, journal citation, and DOI. Funded by SCOAP ${ }^{3}$. temperature and chemical potentials can be defined. An application of anomaly induced transport is the quark gluon plasma created in heavy ion collisions [14]. There the magnetic field is extremely strong. It is also very short lived and might have already decayed before local thermal equilibrium is reached [15]. One therefore needs a better understanding of how anomalies induce transport far out of equilibrium. Holography is an extremely efficient tool to study both anomalous transport phenomena and out of equilibrium dynamics of strongly coupled quantum systems. Previous studies of anomalous transport in holographic quenches focused on the pure $U(1)^{3}$ anomaly [16,17]. This motivates us to study anomalous transport induced by the gravitational anomaly in a holographic quantum quench.

To develop intuition we first consider anomalous hydrodynamics [18-20] in a spatially homogeneous magnetic field. The anomalous transport effect we want to monitor is the generation of an energy current in the magnetic field (2). We start with an initial state at temperature $T_{0}$ and heat the system up to a final temperature $T$. In a relativistic theory the energy current $J_{i}^{\varepsilon}=T_{0 i}$ is the same as the momentum density $\mathcal{P}_{i}=T_{i 0}$. Momentum is a conserved quantity and cannot increase if it is not injected into the system. On the other hand the anomaly induced energy current does increase and this increase must be balanced by a collective flow not included in Eq. (2). The increase in the nondissipative anomalous energy current (2) will be exactly counterbalanced by a dissipative contribution at any given moment. If we introduce a uniform but very light density of impurities carrying some additional charge drag will generate a convective current proportional to the density of impurities [21]. This current measures how much energy current is generated via Eq. (2). We thus need to consider a system with two $U(1)$ charges. The first one has the mixed gravitational anomaly (1) and carries the magnetic flux. The second one is a anomaly free auxiliary $U(1)$ charge 
that serves to monitor the buildup of the current (2) as the temperature changes.

We now consider the hydrodynamics of the system. Since all spatial gradients vanish the constitutive relations are

$$
\begin{gathered}
T_{\mu \nu}=(\varepsilon+p) u_{\mu} u_{\nu}+p \eta_{\mu \nu}+\hat{\sigma}_{B}\left(u_{\mu} B_{\nu}+u_{\nu} B_{\mu}\right), \\
J_{\mu}=\rho u_{\mu}+\sigma_{B} B_{\mu}, \\
J_{\mu}^{X}=\rho_{X} u_{\mu}+\sigma_{B, X} B_{\mu} .
\end{gathered}
$$

The specific forms of the anomalous transport coefficients $\sigma_{B}, \hat{\sigma}_{B}$, and $\sigma_{B, X}$ depend on the hydrodynamic frame choice [23]. In the Landau frame

$$
\begin{gathered}
\hat{\sigma}_{B}=0, \\
\sigma_{B}=24 \alpha \mu-\frac{\rho}{\varepsilon+p}\left(12 \alpha \mu^{2}+32 \lambda \pi^{2} T^{2}\right), \\
\sigma_{B, X}=-\frac{\rho_{X}}{\varepsilon+p}\left(12 \alpha \mu^{2}+32 \lambda \pi^{2} T^{2}\right) .
\end{gathered}
$$

Note that despite $J_{\mu}^{X}$ having no anomaly it does have a nontrivial chiral magnetic transport coefficient in the Landau frame. This makes the effect due to the dragging manifest. We assume that the system is neutral with respect to the anomalous charge, i.e., $\mu=\rho=0$. As the initial condition we take $\vec{J}=\vec{J}^{X}=0$ and the energy current to be given by Eq. (2). Thus, the energy current at any given moment is

$$
32 \lambda \pi^{2} T_{0}^{2} \vec{B}=(\varepsilon+p) \vec{v},
$$

where we work in the linear response regime; i.e., we assume $\lambda B$ to be a small perturbation compared to the energy density and pressure such that $u_{\mu} \approx(1, \vec{v})$. Solving for the velocity $\vec{v}$ and using the constitutive relation for the current $\vec{J}_{X}$ we find that it is given by

$$
\vec{J}_{X}=32 \frac{\rho_{X}}{\varepsilon+p}\left(T_{0}^{2}-T^{2}\right) \pi^{2} \lambda \vec{B} .
$$

This equation determines the current buildup due to drag if the system undergoes a slow near equilibrium time evolution such that an instantaneous temperature $T$ can be defined. It is independent of the choice of the Landau frame. For a generic conformal field theory $p=K T^{4}$ and $\varepsilon=3 p$, and we obtain

$$
j_{X}=\frac{T^{2} / T_{0}^{2}-1}{T^{4} / T_{0}^{4}}
$$

with $j_{X}=\left[\left(K T_{0}^{2}\left|\vec{J}_{X}\right|\right) /\left(8 \pi^{2}\left|\rho_{X} \overrightarrow{\lambda B}\right|\right)\right]$. If a system undergoes near equilibrium evolution $j_{X}$ must lie for any given moment on that curve. Deviation from Eq. (11) will be a benchmark for far from equilibrium behavior.

We will now consider a holographic model that allows us to implement the physics described in a nonequilibrium setting. The action of our model is

$$
\begin{aligned}
S= & \frac{1}{2 \kappa^{2}} \int d^{5} x \sqrt{-g}\left(\mathcal{R}-2 \Lambda-\frac{1}{4} F^{2}-\frac{1}{4 q^{2}} F_{X}^{2}\right. \\
& \left.-\frac{1}{2}(\partial \phi)^{2}+\lambda \varepsilon^{M N O P Q} A_{M S} R_{B N O}^{A} R_{A P Q}^{B}\right) .
\end{aligned}
$$

In addition to gravity and a scalar field $\phi$, it involves two gauge fields $A_{M}$ and $X_{M}$ with field strengths $F=d A$ and $F_{X}=d X$. Only gauge transformations of $A$ are anomalous. The Chern-Simons term is the holographic implementation of the mixed chiral-gravitational anomaly $[6,24]$. We do not include possible pure gauge Chern-Simons terms since we want to isolate the effects of the gravitational anomaly. We set from now on $2 \kappa^{2}=1$.

The dual field theory will be brought out of equilibrium by abruptly varying a coupling. These type of processes are known as quantum quenches [25]. Holographically, the leading mode of bulk fields at the holographic boundary is interpreted as a coupling for the dual field theory [26-28]. We will perform a quench on the coupling associated with the scalar $\phi$. For simplicity, we have chosen $\phi$ to be massless and neutral under the gauge fields $A_{M}$ and $X_{M}$. We consider the following simple boundary profile for its leading mode

$$
\phi_{0}(t, \vec{x})=\frac{1}{2} \eta\left(1+\tanh \frac{t}{\tau}\right),
$$

Since Eq. (12) is invariant under global shifts of $\phi$, the Hamiltonians before and after the quench will be equivalent. The energy density induced by the quench is a function of both its amplitude $\eta$ and time span $\tau$.

In order to be able to match to the hydrodynamic results we will compute the linear response to the magnetic field, which is a valid approximation when $T_{0} \gg \sqrt{B}$. It is thus enough to solve the equations of motion to first order both in the Chern-Simons coupling and the magnetic field. Besides, we will work in the decoupling limit of large charge $q$ for the anomaly free gauge field. Equivalently, we can work at $q=1$ and treat $X^{\mu}$ perturbatively to first order. In that case its dynamics does not backreact onto the other sectors of the theory.

We parametrize the zero-order solution of the metric as

$$
d s^{2}=\frac{1}{z^{2}}\left(-f(t, z) e^{-2 \delta(t, z)} d t^{2}+\frac{d z^{2}}{f(t, z)}+d \vec{x}^{2}\right) .
$$

The equations of motion for the background of gravity and the scalar sector are

$$
\begin{gathered}
\delta=\frac{1}{3} z T_{z z}^{\phi}, \quad f^{\prime}=\frac{4}{z}(f-1)+f \delta, \\
\partial_{t}\left(\frac{e^{\delta} \partial_{t} \phi}{f}\right)=z^{3} \partial_{z}\left(\frac{f e^{-\delta} \partial_{z} \phi}{z^{3}}\right) .
\end{gathered}
$$

These equations are solved numerically using a fourth order Runge-Kutta algorithm. Time reparametrizations are used to fix $\delta(t, 0)=0$, such that the Minkowski metric is 
reproduced at the holographic boundary and the bulk energy momentum tensor of the scalar field is $T_{M N}^{\phi}=$ $\partial_{M} \phi \partial_{N} \phi-\frac{1}{2}(\partial \phi)^{2} g_{M N}$.

Field theories at thermal equilibrium are dual to black hole backgrounds. Our initial geometry will be a Schwarzschild black hole, for which $f=1-\pi^{4} T_{0}^{4} z^{4}$ and $\delta=0$. Around $t=0$ the quench takes place, creating an extra energy density on the boundary theory and, equivalently, a matter shell that enters from the holographic boundary into the bulk. The shell subsequently undergoes gravitational collapse and is absorbed by the original black hole. The resulting black hole represents the final equilibrium state with $T>T_{0}$. Similar holographic setups have been extensively studied in the last years [29-32].

The gauge fields $A_{M}$ and $X_{M}$ satisfy the same equation at zero order in $\lambda$. However, the different roles they should fulfill select different leading solutions. We want $A_{M}$ to induce a constant background magnetic field on the boundary theory and no charge density. Choosing the magnetic field to point in the $x_{3}$ direction, this is achieved by the simple solution $F_{12}=B$ with $B$ constant throughout the bulk. To the contrary, we wish $X_{M}$ to induce a nonvanishing charge density and no boundary field strength, which leads to

$$
F_{X, 0 z}=\rho_{X} z e^{-\delta(t, z)} .
$$

The expectation value of its associated current is given by

$$
J_{X}^{\mu}=\lim _{z \rightarrow 0} \sqrt{-g} F_{X}^{\mu z} .
$$

The integration constant $\rho_{X}$ is the desired charge density.

At linear order in $\lambda$, the magnetic field induces a nondiagonal component in the metric

$$
g_{03}=\frac{4 \lambda B}{z^{2}} \int_{0}^{z} z^{3} e^{-\delta}\left(c+\frac{12}{z^{2}}(f-1)+T_{33}^{\phi}\right),
$$

where $c$ is an integration constant. In the initial and final state the bulk scalar field stress tensor vanishes. We fix the integration constant by demanding that the initial state reproduces Eq. (2), which implies $c=8 \pi^{2} T_{0}^{2}$. In our conventions the energy current can be read off the asymptotic metric expansion as $g_{03}=\frac{1}{4} T_{03} z^{2}+\cdots$ [33].

Neither the scalar field and nor the anomalous gauge field receives a correction at order $\lambda$. However the offdiagonal component of the metric backreacts on $X_{M}$ and generates an entry parallel to the magnetic field. It is governed by the equation

$$
\partial_{t}\left(\frac{e^{\delta} \partial_{t} X_{3}}{z f}\right)-\partial_{z}\left(\frac{e^{-\delta} f \partial_{z} X_{3}}{z}\right)=\rho_{X} \partial_{z}\left(z^{2} g_{03}\right) .
$$

Although $X_{3}$ is sourced by the Chern-Simons term even in the initial state, it leads to a vanishing $J_{X}^{3}$. It is the subsequent evolution that generates a boundary current parallel to the magnetic field. Integrating Eq. (20) in the final state with temperature $T>T_{0}$ we recover Eq. (11) upon normalizing the holographic current (18) accordingly. We also note that due to the fact that $J_{X}^{3}$ is treated in the decoupling limit it reacts immediately to the drag.

We focus first on fast quenches. These are processes whose time span is small in units of the inverse final temperature, $2 \tau T<1$. Figure 1(a) shows the result of two such processes with the same initial temperature $T_{0}$ and time span $\tau$ but different final temperatures. A comparison with the benchmark curve (11), plotted in the lower inset of Fig. 1(a), rules out a possible near equilibrium description. In the hydrodynamic process the current $j_{X}$ attains the maximum when the temperature reaches $T_{m} / T_{0}=\sqrt{2}$, at which it takes the value $1 / 4$. For higher temperatures the equilibrium current decreases, reflecting the large inertia of a hot medium. One of the processes in Fig. 1(a) has been tuned to reach $T_{m}$, while the other generates a higher temperature $T / T_{0}=3$. In both cases $j_{X}$ exhibits a maximum before stabilizing. This contradicts Eq. (11), which would predict a monotonic growth for the process whose final temperature is $T_{m}$, and shows that the evolution is far from equilibrium. Moreover, the current at the maximum is larger than $1 / 4$ in the first quench (blue) and smaller in the second (red), which again disagrees with Eq. (11). When the currents of both processes are normalized to 1 at the final equilibrium state, their profiles in the rescaled time $t T$ are very similar. The current overshoots by around $4 \%$ its final value before attaining it, as can be seen in the upper inset of Fig. 1(a). The time span of a fast quench also has a very small impact on the evolution of the current. This is illustrated in the inset of Fig. 1(b), which shows three processes with the same final temperature $T / T_{0}=2.5$ and a different time span.

It is interesting to compare the evolution of $j_{X}$ with that of another important observable, the energy density. The energy density builds up during the quench and attains its final value as soon as the quench ends. In terms of the
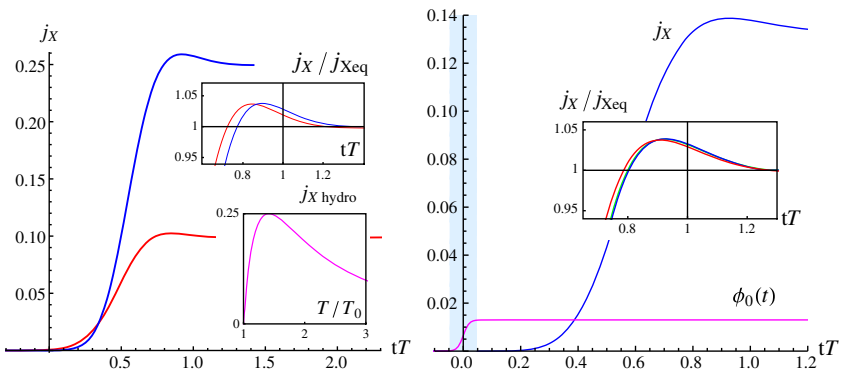

FIG. 1. Left: evolution of $j_{X}$ for quenches with $\tau T_{0}=0.07$ and $T / T_{0}=\sqrt{2}$ (blue) and 3 (red). The final current has been normalized to 1 in the upper inset. The lower inset shows the equilibrium curve (11). Right: very fast quench with $\tau T_{0}=0.007$ and $T / T_{0}=2.5$. The scalar profile (13) is included, shading the time span of the quench. The inset compares this process to two more quenches with $\tau T_{0}=0.0175,0.035$, and the same $T / T_{0}$. 
holographic model the energy pumping into the system happens while the time derivative of the scalar at the holographic boundary is nonvanishing. The profile (13) is within $3 \%$ of its final value at $t / \tau \approx 1.75$. We observe in Fig. 1 that instead $j_{X}$ equilibrates at $t T \approx 1.2$. Hence, the energy density and the current generated in fast quenches have independent equilibration time scales, $\tau$ and $1 / T$, respectively. This provides an alternative and simple way to discard a near equilibrium evolution, since no single effective temperature can describe both observables. At the geometrical level the different equilibration time scales have further implications. While the energy density only depends on the bulk total mass, the anomaly free current must be sensitive to the interior geometry close to the emerging horizon. Consistent with this, $j_{X}$ turns out to mainly build up as equilibrium is approached. The body of Fig. 1(b) describes a very fast quench with $\tau T_{0}=0.007$. The purple curve gives the time profile of the scalar field at the asymptotic boundary (13). The time interval when the quench occurs has been highlighted in blue. Notably, the current is practically zero in this example even sometime after the quench has finished. This clearly shows that the anomaly free current does not react to the initial far from equilibrium state, but to the onset of the equilibrium. A central conclusion of our study is that anomalous transport properties related to the gravitational anomaly are very much linked to the system being at or close to thermal equilibrium.

We wish to now study the transition from fast to slower quenches. Figure 2(a) shows the last stages in the evolution of the current for several processes with the same initial temperature and time span. For the sake of comparison we normalize the final current to 1 , and take the span of the quench instead of the final temperature as the time unit. Processes with $2 \tau T<0.5$ behave as explained above. When $2 \tau T \approx 0.65$ the maximum before equilibration starts to weaken out, and it practically disappears for $2 \tau T \approx 1$. Processes with $2 \tau T \gtrsim 1$ exhibit a monotonic growth of the current to its equilibrium value. We will refer to them as intermediate quenches. We have highlighted the time span of the quench, up to the moment when $\phi_{0}$ is within $3 \%$ of
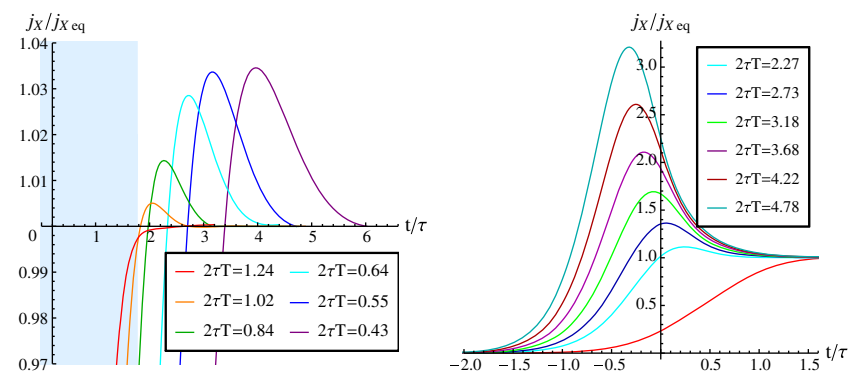

FIG. 2. Left: final approach to equilibrium of $j_{X}$ for several quenches with $\tau T_{0}=0.175$. Right: evolution of $j_{X}$ in processes with $\tau T_{0}=0.175$ and a high final temperature. The red curve coincides on both plots for comparison. its final value. The transition between fast and intermediate quenches happens when the time scale set by the final temperature approaches that of the quench. It is important to stress that, in general, intermediate quenches do not admit either a near equilibrium description. Indeed when the final temperature is higher than $T_{m}$, the absence of a transient maximum signals out of equilibrium dynamics. For the parameters associated with Fig. 2(a) we have $2 \tau T_{m}=0.5$, such that processes with final temperature below $T_{m}$ behave as fast quenches.

Finally, we consider slow quenches. First, we consider processes that are slow with respect to the final temperature and can be expected to have a sizable final period of near-equilibrium evolution. The hydrodynamic current described by the benchmark curve (11) decreases with rising temperature when $T>T_{m}$. In this case, a final period of near-equilibrium evolution implies that $j_{X}$ must exhibit a transient maximum. In Fig. 2(b) we investigate quenches with $2 \tau T>2$ for the same initial conditions as in Fig. 2(a). The red curve coincides in both plots for the sake of comparison. We observe that a maximum reappears with a distinctive characteristic different from that exhibited by fast quenches. Unlike them, the maximum of the quotient $j_{X} / j_{\text {Xeq }}$ increases with temperature, and can be attained even before the quench is halfway through. It is natural that the larger $\tau T$ is the earlier the system enters the nearequilibrium regime, whose onset is qualitatively signaled by the maximum of the current.

In the previous processes $\tau T_{0}=0.175$, such that they are slow with respect to the final temperature but fast with respect to the initial one. This constrains when the near equilibrium regime sets in and hence whether the current can reach 1/4, the maximum of the equilibrium curve (11). Keeping the same $\tau T_{0}$, Fig. 3(a) shows the evolutions with a very high final temperature. Since we are interested in the maximal value of the current, $j_{X}$ has not been rescaled as we did in Fig. 2. Instead of approaching 1/4, the maximum turns out to slowly decrease with increasing final temperature. This shows that the near-equilibrium regime in processes with $\tau T_{0}=0.175$ can only be associated with temperatures well above $T_{m}$. In order for the complete

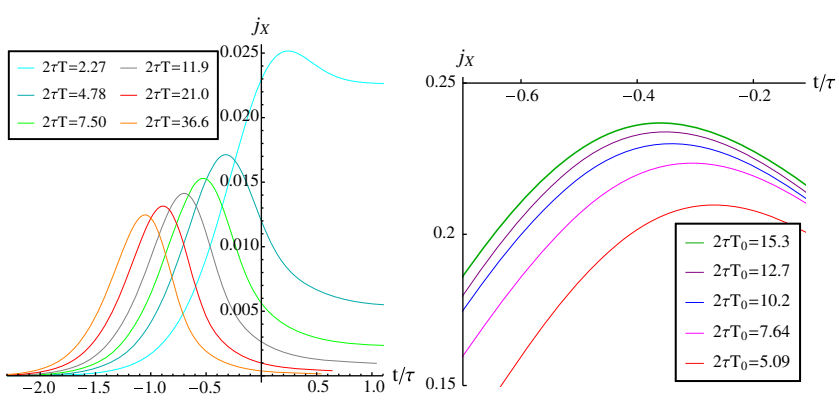

FIG. 3. Left: evolution of $j_{X}$ in processes with $\tau T_{0}=0.175$ and a very high final temperature. Right: processes with fixed initial and final temperatures $T / T_{0}=2.6$ and growing $\tau$. 
evolution to be hydrodynamic, the quench also needs to be slow with respect to the initial temperature. We plot in Fig. 3(b) processes with fixed $T / T_{0}=2.6$ and growing time span. The maximum of the current tends now to $1 / 4$ as expected. We have checked that, consistently, the current builds up in a monotonic way for these slow processes whose final temperature is equal or smaller than $T_{m}$.

We have studied holographic quantum quenches of the $\mathrm{CME}$ induced by the gravitational anomaly. A rich phenomenology arises depending on the time scale of the quench. If the quench is fast with respect to the initial and final temperatures, the evolution is far from equilibrium until the final exponential approach to stabilization. The current builds up late in the time evolution and slightly overshoots before it achieves its equilibrium value. In equilibrium the anomalous conductivity is proportional to the square of the temperature. The main motivation of this work was to analyze what activates the anomalous conductivity out of equilibrium, where there is no notion of temperature. It could have been governed by energy density, which in equilibrium is also measured by the temperature. In this case the current should have reacted as soon as energy is injected into the system. Our result on fast quenches shows that this is not the case. Rather the system has to evolve closer to equilibrium to build up the anomalous current. This is in contrast to the immediate response of other holographic one-point functions, as observed, for example, in Ref. [34].

Intermediate quenches leave the far from equilibrium stage while they are reaching the final state, resulting in a monotonic growth of the current. Processes that are slow with respect to the final temperature but fast with respect to the initial one have a finite period of near-equilibrium evolution. This extends to the complete evolution for large $\tau T_{0}$.

While we have restricted ourselves to the weak field regime appropriate for linear response, it will also be interesting to study the low temperature or high magnetic field regime. Since the buildup of the current requires being close to equilibrium we expect our results to carry over.

We want to thank F. Pena-Benitez for discussions. The work has been supported by Severo Ochoa Programme Grant No. SEV-2016-0597 and by FPA2015-65480-P (MINECO/FEDER).

*karl.landsteiner@uam.es †esperanza.lopez@uam.es *guillermo.milans@csic.es

[1] D. E. Kharzeev, Prog. Part. Nucl. Phys. 75, 133 (2014).

[2] K. Landsteiner, Acta Phys. Pol. B 47, 2617 (2016).

[3] K. Fukushima, D. E. Kharzeev, and H. J. Warringa, Phys. Rev. D 78, 074033 (2008).
[4] A. Vilenkin, Phys. Rev. D 20, 1807 (1979).

[5] K. Landsteiner, E. Megias, and F. Pena-Benitez, Phys. Rev. Lett. 107, 021601 (2011).

[6] K. Landsteiner, E. Megias, L. Melgar, and F. Pena-Benitez, J. High Energy Phys. 09 (2011) 121.

[7] S. Golkar and D. T. Son, J. High Energy Phys. 02 (2015) 169.

[8] D. F. Hou, H. Liu, and H. C. Ren, Phys. Rev. D 86, 121703 (2012).

[9] K. Jensen, R. Loganayagam, and A. Yarom, J. High Energy Phys. 02 (2013) 088.

[10] K. Jensen, R. Loganayagam, and A. Yarom, J. High Energy Phys. 05 (2014) 110.

[11] S. Golkar and S. Sethi, J. High Energy Phys. 05 (2016) 105.

[12] S. D. Chowdhury and J. R. David, J. High Energy Phys. 12 (2016) 116.

[13] J. Gooth et al., Nature (London) 547, 324 (2017).

[14] D. E. Kharzeev, J. Liao, S. A. Voloshin, and G. Wang, Prog. Part. Nucl. Phys. 88, 1 (2016).

[15] V. Skokov, A. Y. Illarionov, and V. Toneev, Int. J. Mod. Phys. A 24 (2009) 5925.

[16] S. Lin and H. U. Yee, Phys. Rev. D 88, 025030 (2013).

[17] M. Ammon, S. Grieninger, A. Jimenez-Alba, R. P. Macedo, and L. Melgar, J. High Energy Phys. 09 (2016) 131.

[18] D. T. Son and P. Surowka, Phys. Rev. Lett. 103, 191601 (2009)

[19] Y. Neiman and Y. Oz, J. High Energy Phys. 03 (2011) 023.

[20] M. A. Stephanov and H. U. Yee, Phys. Rev. Lett. 116, 122302 (2016).

[21] Drag in anomalous fluids has been studied in Refs. [20,22].

[22] K. Rajagopal and A. V. Sadofyev, J. High Energy Phys. 10 (2015) 018.

[23] I. Amado, K. Landsteiner, and F. Pena-Benitez, J. High Energy Phys. 05 (2011) 081.

[24] T. E. Clark, S. T. Love, and T. ter Veldhuis, Phys. Rev. D 82, 106004 (2010).

[25] A. Polkovnikov, K. Sengupta, A. Silva, and M. Vengalattore, Rev. Mod. Phys. 83, 863 (2011).

[26] M. Natsuume, Lect. Notes Phys. 903, 1 (2015).

[27] M. Ammon and J. Erdmenger, Gauge/Gravity Duality: Foundations and Applications (Cambridge University Press, Cambridge, England 2015).

[28] J. Zaanen, Y. W. Sun, Y. Liu, and K. Schalm, Holographic Duality in Condensed Matter Physics, (Cambridge University Press, Cambridge, England, 2015).

[29] P. M. Chesler and L. G. Yaffe, Phys. Rev. Lett. 102, 211601 (2009)

[30] A. Buchel, L. Lehner, and R. C. Myers, J. High Energy Phys. 08 (2012) 049.

[31] J. Abajo-Arrastia, E. da Silva, E. Lopez, J. Mas, and A. Serantes, J. High Energy Phys. 05 (2014) 126.

[32] B. Craps, E. J. Lindgren, A. Taliotis, J. Vanhoof, and H. Zhang, Phys. Rev. D 90, 086004 (2014).

[33] S. de Haro, S. N. Solodukhin, and K. Skenderis, Commun. Math. Phys. 217, 595 (2001).

[34] M. J. Bhaseen, J. P. Gauntlett, B. D. Simons, J. Sonner, and T. Wiseman, Phys. Rev. Lett. 110, 015301 (2013). 